SAND- $-96-8536 C$ RECEIVED

CONF-960835--1

FEB 291996

O\&II

\title{
Commodity Clusters: Performance Comparison Between PC's and Workstations
}

\author{
Russell Carter \\ 1765 Shamrock Ave. \\ Santa Clara, CA 95051 \\ rcarter@geli.com \\ John Laroco \\ Robert Armstrong \\ Sandia National Laboratories \\ Livermore, CA 94551 \\ \{jclaro,rob\}@ca.sandia.gov
}

\section{$1 \quad$ Introduction}

Traditionally, the bulk of large scale scientific and engineering computations were performed on large specialized Supercomputers. In the last seven years, RISC based workstation technology has largely supplanted the Supercomputing market. Using techniques such as workstation clustering, wide classes of problems have been successfully attacked. Workstation clusters are networked RISC UNIX systems commonly provided by vendors such as IBM, Hewlett Packard, SUN, Silicon Graphics, and Digital Equipment Corporation. Each of these vendors is the predominant or proprietary supplier of both hardware and UNIX operating systems.
Applications run on proprietary workstation clusters use UNIX features that enable high performance floating point, fast disk drive transfer rates, and robust networking performance in a multi-user, multi-system environment. UNIX workstation clusters are capable of achieving supercomputer efficiencies on many computationally intensive tasks. Additionally, individual cluster systems can be used as desktop UNIX workstations.

The size of the PC market is about nine times larger than the proprietary UNIX workstation market. Intel Pentium based systems are the performance leader in this market. Until recently, proprietary

workstation hardware and software greatly exceeded 
in sophistication, capability, and performance that available from the commodity PC distribution channel. Recent PC technology advances have dramatically increased processor, main memory and cache memory performance. Some high end models offered by proprietary workstation vendors still maintain a significant advantage in peak floating point performance. However, the widespread availability of numerous high performance $\mathrm{PCI}$ bus network, video, and disk controllers for PCs has erased the traditional hardware $\mathrm{I} / \mathrm{O}$ performance advantage of proprietary UNIX workstations. A similar evolution has occurred in system software. The multi-user performance of several varieties of PC UNIX running on Intel Pentium CPUs is equivalent to proprietary workstation UNIX. The enormous size of the commodity PC market ensures lowest possible hardware costs. Other advantages exist: individual commodity workstations are well engineered PCs, and may be maintained by any competent PC maintenance vendor. Organizations have more options to manage computer resources efficiently, since every commodity workstation can run industry standard operating systems, such as Microsoft Windows 95, Windows NT, IBM OS/2, and SunSoft's Solaris.
With these considerations in mind, in 1994 the Distributed Computing Research group at Sandia National Laboratories, CA, constructed a testbed of sixteen Pentium workstations. Dubbed the DAISy (Distributed Array of Inexpensive Systems), this testbed was used to investigate the viability of commodity workstation clusters. Extensive functionality, performance and cost studies have been compared with performance and cost data from the proprietary workstation vendors. Full UNIX operating system functionality is provided by the BSD 4.4 based OS. The advanced networking applications required to manage clusters of workstations were found to be robust and full featured. Performance of $I / O$ subsystems is equivalent or superior. Floating point performance is equivalent or superior to low-end offerings from the traditional workstation vendors. Initial and ongoing costs for installing and operating a commodity workstation cluster are about one half (50\%) that of similar functional configurations from the traditional workstation vendors.

\section{$1.1 \quad$ Related Work}

A commodity workstation cluster project using similar technology is the Beowulf project [1]. The emphasis of this project is on lowest possible cost components combined with striped $10 \mathrm{Mb}$ Ethernet. 


\section{DISCLAIMTER}

Portions of this document may be illegible in electronic image products. Images are produced from the best available original document. 
Good results have been obtained for important

parallel codes. Scalability has been limited because

each PC in the cluster share the same $10 \mathrm{Mbit} / \mathrm{sec}$

Ethernet.

\section{DAISy Node Configuration}

The DAISy Cluster is dual homed network of 16 Intel Pentium 90MHz workstations (see Figure 1) and very inexpensive UNIX compatible software. DAISy is a homogeneous research prototype used for scientific parallel distributed computing, and, a model for a minimum cost and fast distributed computational system. The motherboards support 3 PCI (Peripheral Component Interface)[2] bus cards and 4 ISA (Industrial Standard Architecture) bus cards. Each node has $256 \mathrm{Kbytes}$ of 2 nd level cache and 64Mbytes of random access memory (RAM).

The PCI bus is noteworthy for the reason that it is the first high performance, asynchronous $1 / O$ bus available for commodity PC architectures. Disk I/O functionality is handled by a bus-mastering PCI SCSI-II controller.

Each node in the model consists of :

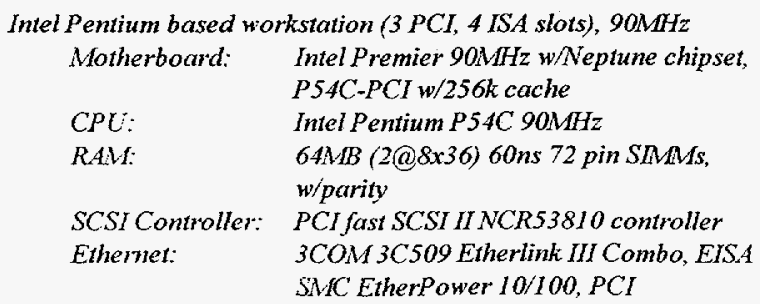

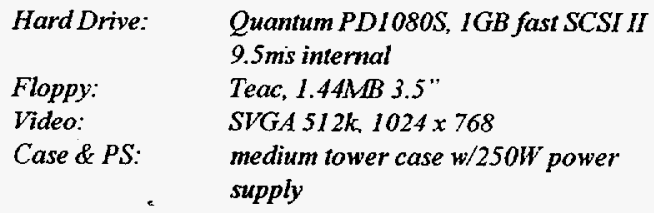

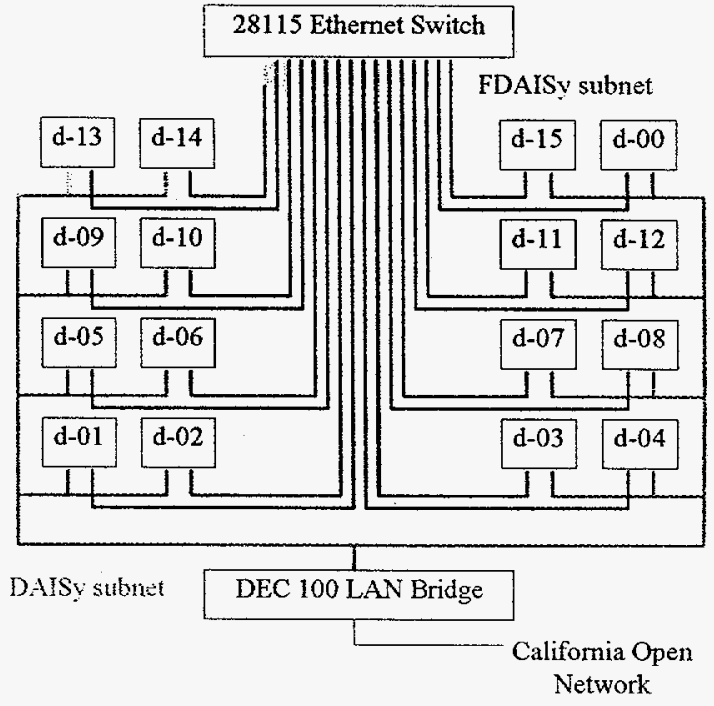

Figure 1. DAISy \& FDAISy Subnet. Individual CPU's are labeled d-00 through d-15.

\subsection{The P54C Pentium ${ }^{\mathrm{TM}} 90 \mathrm{MHz}$ Processor} In the early 90 's, the installed large scale computing resources at Sandia National Lab, CA, evolved to a cluster of RISC UNIX systems. With free UNIX compatible software already available on architectures based on the $1386^{\mathrm{TM}}$ and $\mathrm{i} 486^{\mathrm{TM}}$ CPUs the Distributed Computing Research Group at SNL, CA decided to construct a network of commodity components based on the $x 86 \mathrm{CPUS}$, the P54C Pentium ${ }^{\mathrm{TM}}$ Processor was the logical choice. 
The P54C (90 MHz Pentium) was the state-of-theart commodity PC CPU at the time of initial construction of DAISy. At the time of this writing, $166 \mathrm{MHz}$ Pentiums and $200 \mathrm{MHz}$ Pentium Pros are standard, leading to CPU performance increases of a factor of 2-4. Advances in cache memory and main memory technology have increased performance of these critical components by over $50 \%$. The measured performance of the P54C-90 is used as the basis for the computation of price/performance.

\section{$2.2 \quad$ Network}

The networks hosted on each node of the cluster are standard 10Mb/s ISA bus (10BASE-2) Ethernet and switched $100 \mathrm{Mb} / \mathrm{s}$ PCI bus (100BASE-TX) Fast Ethernet. The 10BASE-2 network is a bus broadcasting network topology. This interface is used for client node NFS mounts, and any client node interactive work users find necessary. The 100BASE-TX network facility uses a high speed frame switch (28115 Fast Ethernet Switch by Bay Networks) with PCI bus fast Ethemet (10/100BaseT) NICs connected in a point-to-point star topology. The designated use of the 100BASE-TX network is for user program message passing traffic. Hence, the architecture of the 100Base-TX network was designed to ensure contention free, high performance network communications. DAISy is a subnet on Sandia's Internet backbone via a DEC 100 bridge. The choice of networks was dictated by the architecture of the motherboards available at the time of design; the Intel motherboards used do not support more than one bus-mastering network interface card.

\subsection{Frame Switched 100BASE-TX Fast Ethernet}

The 100BASE-TX Fast Ethernet Network uses a Synoptics 28115 Frame Switch to reduce latency and increase aggregate bandwidth available to message passing functions in user programs on the DAISy cluster. Frame switching is used to enhance network performance by increasing the total amount of available aggregate bandwidth and decreasing overall communication latency. In the case of DAISy, an increased bandwidth of a factor of ten increases the number of parallel applications suitable for the DAISy cluster. Bandwidth is increased because contention is eliminated and multiple transmissions are allowed. For instance, ordinary shared media broadcast through a type of pipe communication. That is, all nodes connected to that pipe can see the broadcast, therefore; (1) all nodes look at the broadcast frame, (2) decide if the frame belongs to them, and. (3) act accordingly if the frame was addressed to them, otherwise (4) the 
nodes just continue to monitor the pipe. The advantage of a frame switch is frames are unicast only to the port attached to the destination, much like the crossbar interconnect network seen in multiprocessor machines. Because the frame is only transmitted on a single port, other ports are available for other simultaneous transmissions Frame Frame

\section{DAISy System Software Configuration}

\section{$3.1 \quad$ Operating System}

The operating system is the freely redistributable FreeBSD [3], a BSD 4.4Lite-Derived UNIX OS.. The choice of OS was made on the basis of support for high performance PCI devices, performance of device drivers on the high performance PCI network and disk drive devices, performance of NFS server and client services, availability of system source code, and overall cost. Interestingly, no commercial OS approaches the thoroughness by which FreeBSD satisfied the requirements. Linux [4] is an obvious possibility (and was the initial OS) but was replaced due to lack of adequate PCI device support, PCI device driver performance, and NFS server performance.

The directory hierarchy is a common for workstation clusters. Users home directories and the various local "/usr/local" applications are installed on the main drive of the master node, which functions as an NFS server to the remaining 15 client nodes.

\subsection{Message Passing Software}

The goal of the DAISy is to provide the highest possible price performance using commodity hardware and software resources. The parallel architecture of DAISy requires a user accessible means of parallel programming. The 100BASE-TX network is used solely for user application messagepassing traffic. The 10BASE-2 network is used for NFS and system uses. Message passing libraries available to the DAISy users are PVM3[5] and MPI (MPICH)[6].

\section{$4 \quad$ System Analysis}

A goal of the DAISy project is to investigate the viability of commodity PC technology to the computation of scientific and engineering problems traditionally performed on "Supercomputers", and more recently high performance RISC workstations and clusters of RISC workstations. To this end a performance analysis of the various subsystems was carried out. Finally, performance of the cluster as a whole on a number of parallel applications was determined. The results are given in the following sections. 
The compiler used for all performance tests is gcc-

\subsubsection{FORTRAN code is translated to $\mathrm{C}$ using a}

translator and then compiled using gcc-2.6.3. This

particular version of gcc did not support any

Pentium specific optimizations, which are becoming common in commercial compiler products.

\begin{tabular}{|llcccc|}
\hline $\begin{array}{l}\text { System } \\
\text { Description }\end{array}$ & $\begin{array}{l}\text { Operating } \\
\text { System }\end{array}$ & CPU & MHz & Year & $\begin{array}{c}\text { List } \\
\text { Price }\end{array}$ \\
\hline \hline FreeBSD/586 & FreeBSD & Pentium 90 & 90 & 994 & $5 \mathrm{~K}$ \\
(p5-90) & 2.1 & & & & \\
DEC Alpha & OSF1 3.2 & Alpha & 175 & 992 & $24.4 \mathrm{~K}$ \\
HP 9000/735 & HP-UX & PA-RISC & 99 & 9 & $33 \mathrm{~K}$ \\
& A.09.05 & & & & \\
IBM RS6000 & AIX 2.3.5 & RS6000 & 40 & 992 & $29 \mathrm{~K}$ \\
SGI IRIX & IRIX 5.3 & MIPS R4000 & 100 & 992 & $33 \mathrm{~K}$ \\
SUN SS10, & SUNOS & SUper & 51 & $' 92$ & $18.9 \mathrm{~K}$ \\
SUN/TI & 4.1 .3 & SPARC & & & \\
\hline
\end{tabular}

Table 1. DAISy and HEAT Cluster system descriptions.

For comparison, there are various other system

performance results shown throughout the paper.

One such system configuration is another cluster at

SNL, CA, HEAT (Heterogeneous Environment And

Testbed). The HEAT cluster is a collection of five

flavors of mainstream workstations. There are 50

workstations connected together to form HEAT, 10

each of the following: SUN SS10, SGI R4000

Indigo, DEC Alpha, HP 735, and IBM RS6000 350.

The network media for HEAT is a bridging crossbar gigaswitch with 22 FDDI ports. Table 1 shows a description of the DAISy and HEAT clusters. A note as to where the other system performance results were obtained will be given in the respective subsections.

\subsection{Subsystem Performance}

The performance of a workstation cluster is strictly limited by the performance of applications on each of the individual nodes. The individual node performance is in turn a function of how efficient the important subsystems perform their tasks. For the class of scientific and engineering applications of interest the individual node performance measure most often quoted is the number of millions of floating point operations performed per second, or MFLOPS. The most important subsystems that determine individual node floating point performance are Main Memory and the CPU.

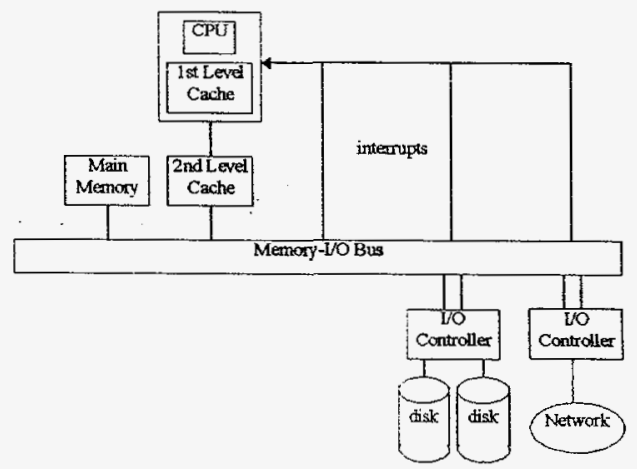

Figure 2. Typical collection of I/O devices on a computer.

The I/O subsystems of interest are disk and networking. The disk $1 / O$ performance is characterized by bandwidth through the file system, and is important for those applications that use local 
storage. Network File System (NFS) performance is crucial for efficient operation of the workstation cluster. Both client and server performance of NFS is important, the first in order to implement efficient distribution of executables at user program startup, and the latter to allow timely transferal of computed data to the user's home directory on the master node. Finally, networking performance is characterized by bandwidth and latency, and affects the performance of parallel message passing algorithms on the cluster. Figure 2 shows a typical collection of $\mathrm{U} / \mathrm{O}$ devices which are of interest when determining system performance.

\subsection{Floating Point performance}

The performance of the Pentium CPU on floating point is discussed in detail in [7],[8]. The following discussion, and performance measurements, are for 64 bit floating point operations. The Pentium CPU has a single 8 stage pipeline for floating point operations, with a capability of producing one result per cycle, so the "not-to-be-exceeded" performance is 1 FLOP per cycle, or 90 MFLOPS for the $90 \mathrm{MHz}$ P54C. However, the P54C architecture uses a stack of floating point registers rather than an independently addressable register set. A common operation is to swap operands within the stack. When paired with certain floating point operations this swap can happen simultaneously, so that no cycles are lost. This cannot be done in every case. The most highly tuned assembly coded kemels achieve a maximum floating point performance of roughly 2/3 FLOP per cycle, or 60 MFLOPS for the $90 \mathrm{MHz}$ P54C.

\subsubsection{Matrix-Matrix multiply (using assembly coded DGEMM)}

Matrix-Matrix multiply is a highly optimized kernel computation which, when well implemented, is for practical purposes an indicator of the upper bound of the floating point performance available to user applications on a single node. The performance figures provided here use the BLAS3 [9] DGEMM algorithm. The BLAS 3 routines are generally provided as tuned assembly coded routines by RISC UNIX workstation vendors. The P54C-90 Pentium results were obtained using a tuned version of the DGEMM algorithm, using assembly coded DAXPY routines implemented by one of the authors. The best performance obtained was 13.3 MFLOPS on 64x64×64 DGEMM. Properly tuned DGEMM implementations allow for the CPU to operate out of the highest level of the memory hierarchy, in this case, the first level cache. 


\subsection{Main Memory Performance}

Commodity PC architecture is similar to low and mid-class RISC workstation architecture: The processor is fed operands from a series of memory hierarchies, which generally differ in speed, cost, and size. Competitive pressures require that the fast memory hierarchies (1st and 2nd level cache) be made as small as feasible, and thus many applications of interest have the characteristic that a significant amount of data is fetched relatively frequently from the large (and slow) main memory. If main memory is too slow, the performance of many applications will be limited by the speed with which memory can supply operands.

Prior to the availability of Fast Page Mode DRAM, commodity PC main memory was significantly slower than that available for most RISC UNIX workstations. Since implemented in the first quarter of 1994, Fast Page Mode DRAM has become the memory of choice for commodity PCs. Now commodity PCs exhibit main memory bandwidths that compare favorably to that of RISC workstations offered by the traditional workstation vendors.

\subsubsection{Stream benchmark}

The most widely used measure of main memory bandwidth is McCalpin's Stream [10] benchmark. This benchmark performs a carefully parameterized
DAXPY, and returns information on several aspects of main memory performance. A wide range of results are available. The benchmark is notable because it emphasizes the measurement of the rate at which operands can be fetched to the CPU from the lowest level of the memory hierarchy, which for most workstation class systems is the large DRAM based main memory. Table 2 shows the results from both the DAISy and HEAT clusters.

\begin{tabular}{|c|c|c|c|c|}
\hline Func & $\begin{array}{c}\mathrm{MB} / \mathrm{s} \\
\mathrm{p} 5-90\end{array}$ & $\begin{array}{c}\text { DEC } \\
\text { Alpha }\end{array}$ & $\begin{array}{c}\text { IBM } \\
\text { RS6K }\end{array}$ & $\begin{array}{c}\text { SGI } \\
\text { IRIX }\end{array}$ \\
\hline$\overline{\text { Assi }}$ & 38.908 & 88.148 & 125.912 & 39.903 \\
\hline & 39.178 & 88.83 & 122.966 & 37.597 \\
\hline Summing & 46.03 & 93.155 & 130.42 & 38.856 \\
\hline SAXPYing & 46.032 & 91.699 & 130.285 & 36.285 \\
\hline
\end{tabular}

Table 2. Stream results (MB/s).

\subsubsection{Imbench benchmark (Memory Bandwidth)}

The lmbench [11] suite measures the ability to read, and write data over a varying set of sizes. The benchmarks included in the memory bandwidth component include: $b_{-} w_{-} m e m$ rd and bw_mem_wr. The results shown in table 3 are from the DAISy and HEAT systems and from McVoy and Staelin's Imbench draft.

Memory reading bandwidth is measured by an unrolled loop that sums up a series of integers (typically a 4 byte integer). The benchmark bw_mem_rd allocates the specified amount of 
memory, zeros it, and then times the reading of the memory as a series of integer loads and adds. An $8 \mathrm{MB}$ area is specified in Table 3 to show memory bandwidth and not cache bandwidth.

Memory writing bandwidth is measured by an unrolled loop that stores a value into an integer (typically a 4 byte integer) and then increments the pointer. The benchmark bw_mem_wr allocates the specified amount of memory, zeros it, and then times the writing of that memory as a series of integer stores and increments. Again, an 8MB area is specified in Table 3 to show memory bandwidth and not cache bandwidth.

\begin{tabular}{|c|c|c|}
\hline System & $\begin{array}{l}\text { memory } \\
\text { read (bw_mem_rd) }\end{array}$ & write (bw_mem_wr) \\
\hline IBM Power2 & 205 & 364 \\
\hline Sun Ultra 1 & 129 & 152 \\
\hline DEC Alpha@300 & 120 & 123 \\
\hline $\mathrm{HP}$ K210 & 117 & 126 \\
\hline Unixware/i686 & 214 & 86 \\
\hline DECAlpha@150 & 79 & 91 \\
\hline Linux/1686 & 208 & 56 \\
\hline Linux/Alpha & 73 & 71 \\
\hline FreeBSD/1586 & 73 & 83 \\
\hline Linux/Alpha & 73 & 71 \\
\hline Linux/i586 & 74 & 75 \\
\hline SGI Challenge & 65 & 67 \\
\hline SGI Indigo & 69 & 66 \\
\hline IBM PowerPC & 63 & 26 \\
\hline Sun SC1000 & 38 & 31 \\
\hline \multicolumn{3}{|l|}{ DAISy systems } \\
\hline FreeBSD/i586 (p5-90) & 54.15 & 28.01 \\
\hline \multicolumn{3}{|l|}{ HEAT systems } \\
\hline DEC Alpha & 84.8 & 90.31 \\
\hline HP $9000 / 735$ & 52.81 & 50.88 \\
\hline IBM RS6000 & 59.03 & 61.32 \\
\hline SUN SSIO & 42.6 & 28.18 \\
\hline SGI IRIX & 44.47 & 48.94 \\
\hline
\end{tabular}

Table 3. bw_mem_rd, bw_mem_wr results (MB/s).
SPECint92 and SPECfp92 results showing the top 20 SPECx92 performance ratings as reported at the URL, htp://www.ideas. comaubench/spec/spec.html, and the results of the P5-90 and P6200 from URL http:/hpwww.epfl.ch/bench/SPEC.html.

\begin{tabular}{|lcc|}
\hline System & SPECint & SPECfp \\
Name & 92 & 92 \\
\hline \hline DEC-AlphaServer $82005 / 300$ & 341.4 & 512.9 \\
DEC-AlphaServer 8400 5/300 & 341.4 & 512.9 \\
Olivetti-LSX 7830 & 341.4 & 512.9 \\
Olivetti-LSX 7860 & 341.4 & 512.9 \\
DEC-AlphaStation 600 5/300 & 337.8 & 502.1 \\
Sun-Ultra 2 Model 2200 & 332 & 505 \\
DEC-AlphaStation 600 5/266 & 289 & 405 \\
DEC-AlphaServer 2000 5/250 & 277.1 & 410.4 \\
Olivetti-LSX 7560 & 277.1 & 410.4 \\
DEC-AlphaServer 2100 5/250 & 277 & 410.4 \\
Sun-Ultra 1 Model 170 & 252 & 351 \\
Sun-Ultra 1 Model 170E & 252 & 351 \\
Sun-UltraServer 1 Model 170 & 252 & 351 \\
Sun-UltraServer 1 Model 170E & 252 & 351 \\
Sun-Ultra 1 Model 140 & 215 & 303 \\
Sun-UltraServer 1 Model 140 & 215 & 303 \\
HAL-HALstation 350 & 212 & 271 \\
HAL-HALstn 350 Application Svr & 212 & 271 \\
DEC-AlphaServer 2000 4/275 & 202.9 & 292.6 \\
DEC-AlphaServer 2100 4/275 & 202.9 & \\
\hline Intel Xpress Pentium 60/90 512+8/8 & $\mathbf{1 0 6 . 5}$ & $\mathbf{8 1 . 4}$ \\
Intel Alder PentiumPro 200 256+8/8 & 366 & 283.2 \\
\hline
\end{tabular}

Table 4. SPECint92 and SPECfp92 performance ratings.

\subsubsection{SPECx92: Standard Performance Evaluation Corporation}

The Standard Performance Evaluation Corporation (SPEC) [12] was founded in, 1988, as a non-profit group of computer vendors, system integrators, universities, research organizations, publishers and consultants throughout the world. It was formed with the objective to establish, maintain and endorse a standardized set of relevant benchmarks that can be applied to the newest generation of highperformance computers. The SPEC 92 benchmarks are the second generation of the SPEC benchmarks. A third major version is the SPEC95 suite. 
Unfortunately, insufficient results were reported for systems that resemble DAISy systems for the SPEC95 suite. Shown in Table 4 are the top 20 SPECx92 performance ratings and, Intel P5 $90 \mathrm{MHz}$ and P6 200MHz systems.

\subsubsection{LINPACK (C and FORTRAN)}

LINPACK[13] is a venerable floating point benchmark that primarily demonstrates performance on a very simple set of loops. Code is provided on the NETLIB[14] software repository for both C and FORTRAN versions.

Performance on the $\mathrm{C}$ version is somewhat better, as is expected from the need to translate FORTRAN code first to $\mathrm{C}$ before compilation. However, FORTRAN performance is surprisingly good, and well within the competitive range of higher priced offerings from the traditional RISC Workstation vendors. Results in Table 5 and Table 6 are from DAISy and HEAT systems.

\begin{tabular}{|l|cccccc|}
\hline linpacke & p5-90 & $\begin{array}{c}\text { DEC } \\
\text { Alpha }\end{array}$ & $\begin{array}{c}\text { HP } \\
735\end{array}$ & $\begin{array}{c}\text { IBM } \\
\text { RS6K }\end{array}$ & $\begin{array}{c}\text { SGI } \\
\text { IRIX }\end{array}$ & $\begin{array}{c}\text { SUN } \\
\text { SS10 }\end{array}$ \\
\hline MFLOPS & 7.4 & 19.3 & 18.7 & 12.6 & 8.1 & 10.1 \\
\hline
\end{tabular}

Table 5. LINPACK C results.

\begin{tabular}{|c|ccccc|}
\hline linpackd & p5-90 & $\begin{array}{c}\text { DEC } \\
\text { Alpha }\end{array}$ & $\begin{array}{c}\text { HP } \\
735\end{array}$ & $\begin{array}{c}\text { IBM } \\
\text { RS6K }\end{array}$ & $\begin{array}{c}\text { SGI } \\
\text { IRIX }\end{array}$ \\
\hline MFLOPS & 6.151 & 9.909 & 5.607 & 4.862 & 4.86 \\
\hline
\end{tabular}

Table 6. LINPACK FORTRAN results.

\subsubsection{Discussion}

As seen from the results, the main memory

bandwidth of the $90 \mathrm{MHz}$ Pentium is well within that of many low-end workstations offered by the traditional workstation vendors. This result indicates that many applications that depend on the performance of main memory, can be expected to perform well, provided the speed of the CPU is sufficient. Also, note the significant gain in main memory bandwidth from the $90 \mathrm{MHz}$ Pentiums to the 133MHz Pentiums and to the now standard Pentium Pro's. This indicates that with little additional investment DAISy can be upgraded with a significant increase in performance.

\subsection{Disk Performance}

Many interesting applications require significant amounts of local DISK I/O capability.

\subsubsection{Bonnie: Disk performance benchmark}

The Bonnie[15] disk performance benchmark measures several aspects of disk performance. Table 7 shows the DAISy and HEAT disk performance benchmarks for sequential output, sequential input, and random seeks on a per character and a block size measure. The benchmark size was $100 \mathrm{MB}$. 


\begin{tabular}{|c|c|c|c|c|c|c|c|c|c|c|c|c|c|}
\hline \multirow[b]{2}{*}{ Machine } & \multirow[b]{2}{*}{ MB } & \multicolumn{6}{|c|}{ Sequential Output } & \multicolumn{4}{|c|}{ Sequential Input } & \multicolumn{2}{|c|}{ Random } \\
\hline & & $\begin{array}{l}\mathrm{Per} \text { Ch } \\
\mathrm{K} / \mathrm{sec}\end{array}$ & $\%$ сpu & $\begin{array}{l}\text { Block } \\
\mathrm{K} / \mathrm{sec}\end{array}$ & $\%$ сpu & $\begin{array}{c}\text { Rewrite } \\
\mathrm{K} / \mathrm{sec}\end{array}$ & $\%$ ocpu & $\begin{array}{l}\text { Per Cha } \\
\mathrm{K} / \mathrm{sec}\end{array}$ & \%срu & \begin{tabular}{|c} 
Block \\
K/sec
\end{tabular} & $\%$ сри & $\begin{array}{r}\text { Seeks } \\
/ \mathrm{sec}\end{array}$ & $\%$ сpu \\
\hline$\overline{\text { P5-90 }}$ & 100 & 2059 & 93.6 & 2429 & 57.6 & 971 & 18.7 & 1302 & 49.6 & 2280 & 29.9 & 156.5 & 13.9 \\
\hline DEC Alpha & 100 & 3425 & 95.6 & 3491 & 14.5 & 1538 & 6.8 & $3497^{\circ}$ & 96.9 & 3574 & 8.6 & 101.6 & 3.6 \\
\hline HP 735 & 100 & 1568 & 60.6 & 1492 & 31.7 & 610 & 4.5 & 1492 & 56.9 & 1530 & 7.5 & 141 & 6 \\
\hline IBM RS6K & 100 & 1459 & 96.5 & 1558 & 13.3 & 530 & 6.4 & 1123 & 89.2 & 1939 & 13.2 & 44.8 & 6 \\
\hline SGI IRCX & 100 & 1767 & 95.4 & 3307 & 26.5 & 1320 & 13.5 & 1336 & 81.6 & 2806 & 15.8 & 62.9 & 5 \\
\hline SUN SS10 & 100 & 1503 & 71.7 & 1552 & 15.4 & 523 & 8.4 & 1415 & 81.9 & 2275 & 20.5 & 71.9 & 6.6 \\
\hline
\end{tabular}

Table 7. Bonnie results (100MB benchmark size).

\begin{tabular}{|c|c|c|c|c|c|c|c|c|c|c|c|c|c|}
\hline \multirow[b]{2}{*}{ Machine } & \multirow[b]{2}{*}{ MB } & \multicolumn{6}{|c|}{ Sequential Output } & \multicolumn{4}{|c|}{ Sequential Input } & \multicolumn{2}{|c|}{ Random } \\
\hline & & $\begin{array}{l}\text { Per Cha } \\
\text { K/sec }\end{array}$ & \%рu & $\begin{array}{c}\text { Block } \\
\mathrm{K} / \mathrm{sec}\end{array}$ & $\%$ срu & $\begin{array}{l}\text { Rewrite } \\
\mathrm{K} / \mathrm{sec}\end{array}$ & $\%$ \%рu & $\begin{array}{l}\mathrm{Per} \text { Cha } \\
\mathrm{K} / \mathrm{sec}\end{array}$ & $\%$ \%pu & $\begin{array}{c}\text { Block } \\
\mathrm{K} / \mathrm{sec}\end{array}$ & $\%$ срu & $\begin{array}{r}\text { Seeks } \\
/ \mathrm{sec}\end{array}$ & \%срu \\
\hline P5-90 & 100 & 131 & $\overline{4.5}$ & 128 & $\overline{1.1}$ & 12 & $\overline{0.3}$ & 660 & 24.4 & 571 & 4.7 & 25.4 & 3.8 \\
\hline DEC Alpha & 100 & 519 & 13.6 & 756 & 3.4 & 350 & 1.7 & 1065 & 27 & 1201 & 2.6 & 99.2 & 5.2 \\
\hline HP 735 & 100 & 645 & 25.4 & 679 & 11.9 & 330 & 2.3 & 835 & 31.6 & 1032 & 4.9 & 106.6 & 4.8 \\
\hline IBM RS6K & 100 & 562 & 38.9 & 692 & 8.2 & 289 & 5 & 405 & 33.5 & 1440 & 11.6 & 35.4 & 2.9 \\
\hline SGI IRIX & 100 & 258 & 16.6 & 287 & 3.4 & 188 & 6 & 174 & 14.4 & 425 & 7.8 & 26.6 & 5.8 \\
\hline SUN SS10 & 100 & 407 & 20.5 & 581 & 8.1 & 252 & 6 & 463 & 27.5 & 615 & 6.3 & 47 & 8.3 \\
\hline
\end{tabular}

Table 8. Bonnie NFS Server Performance results (25MB benchmark size).

\subsubsection{NFS Server Performance}

NFS server performance (see Table 8 ) was

assessed by running the bonnie benchmark on a

client node to the user home directory file

system on the master node, using the DAISy

10BASE-2 operational network.

\subsubsection{NFS Client Performance}

Client performance was measured by copying a

large file from the master node to $/ \mathrm{dev} / \mathrm{null}$ on

the client system. For an $11 \mathrm{MB}$ file the best

rate was measured at $602 \mathrm{~KB} / \mathrm{s}$

\subsubsection{Discussion}

The results from the bonnie benchmark show

that the DAISy cluster systems have disk

performance equivalent or superior to that of the measured HEAT systems. The NFS server

performance shows the disadvantage of the

current Intel motherboards inability to support

more than one bus-mastering network interface

card.

\subsection{Networking Bandwidth and Latency}

The DAISy project's first stage interconnect

transport protocol for the message passing

libraries is TCP (with RouteDirect PVM3

operations). The performance of the DAISy

cluster as a whole on parallel message passing

applications then is at minimum bounded by the

underlying TCP performance of the 100BASE-

TX interconnect. Two aspects of the switched

100BASE-TX network directly affect the 
performance of parallel message passing applications. In the simplest case two nodes communicate, in point-to-point fashion. The second, more complex case occurs when more than two nodes wish to communicate contemporaneously. The performance analysis of this important case is much more complex and is beyond the scope of this paper. In the point-to-point case, two common measures of TCP performance are bandwidth and latency. Bandwidth is defined as the asymptotic number of bytes transferred per unit time, as the size of messages size is increased. Latency is defined to be the extrapolated zero byte message transfer time obtained from a linear fit of a range of (small) message sizes. A useful and widely used tool to determine these quantities at the TCP socket level is Netperf.[16]. These network performance results were confirmed using the lmbench benchmark suite. Direct measures of the bandwidth and latency of point to point message passing latency and bandwidth PVM3 and MPI libraries were also performed.

\subsubsection{Netperf suite: A Network Performance Benchmark}

Netperf is a suite of benchmarks used to measure various aspects of networking performance and designed around the client/server model. The primary focus is on bulk data transfer and request/response performance using either TCP or UDP and the Berkeley Sockets interface. All benchmarks are run for an elapsed time of $\sim 60$ seconds. The various netperf performance benchmarks fall into two categories: (a) stream, and (b) request/response.

The most common use of the netperf suite is measuring bulk data transfer performance. This is referred to as "stream" or "unidirectional stream" performance. These tests measure how fast one system can send data to another and/or how fast that other system can receive it. The "tcp_stream_script" and "udp_stream_script" shell scripts supplied with the package were run to determine bandwidth..

Netperf request/response performance is quoted as "transactions/sec" for a given request and response size. A transaction is defined as the exchange of a single request and a single response. From a transaction rate, one can infer one way and round trip average latency. The "request/response" scripts that were run include: tcp_rr_script and udp_rr_script. 
TCP Stream: tcp_stream_script is an

implementation of the stream benchmark over

TCP. The local send size ranges from 4096 to

32768 bytes with the local/remote send and

receive socket buffer sizes of each ranging from

8102 to 57344 bytes. With the send and receive

socket buffer sizes not remaining constant, the

output shows throughput $(\mathrm{Mb} / \mathrm{s})$ as a function of range (bytes). For the results in Table 9 recv \& send socket size $=57344$, send message size $=$ 32768.

\begin{tabular}{|c|c|c|c|c|}
\hline System & Network & $\begin{array}{l}\mathrm{TCP} \\
\text { stream }\end{array}$ & $\begin{array}{r}\text { UDP } \\
\text { stream } \\
\text { send }\end{array}$ & receive \\
\hline $\begin{array}{l}\text { DAISy systems } \\
\text { FreeBSD/i586 (p5-90) } \\
\text { FreeBSD/1586 (p5-90) }\end{array}$ & $\begin{array}{c}\text { 10base } 2 \\
\text { 100baseT }\end{array}$ & $\begin{array}{c}5.19 \\
50.64\end{array}$ & $\begin{array}{l}5.73 \\
68.5\end{array}$ & $\begin{array}{c}5.73 \\
35.28\end{array}$ \\
\hline $\begin{array}{l}\text { HEAT systems } \\
\text { DEC Alpha } \\
\text { HP } 9000 / 735 \\
\text { SGI IRDX }\end{array}$ & $\begin{array}{l}\text { fddi } \\
\text { fddi } \\
\text { fddi }\end{array}$ & $\begin{array}{l}85.87 \\
79.31 \\
71.08\end{array}$ & $\begin{array}{l}89.23 \\
87.38 \\
69.66\end{array}$ & $\begin{array}{l}37.14 \\
52.18 \\
11.45\end{array}$ \\
\hline
\end{tabular}

Table 9. tcp_range_script, tcp_stream_script, udp_stream_script results $(\mathrm{Mb} / \mathrm{s})$.

UDP Stream: udp_stream_script is an

implementation of the stream benchmark over

UDP. The difference between udp_stream and

tcp_stream is that the send size cannot be larger

than the smaller of the local and remote socket

buffer sizes. The local send size ranges from 64

to 1472 bytes with the local/remote send and

receive socket buffer sizes of each remain

constant at 32768 bytes. With the send and receive socket buffer sizes remaining constant, the output shows throughput $(\mathrm{Mb} / \mathrm{s})$, as a function of range (bytes) for both send and receive. For the results inTable 9: socket size $=$ 32768 , message size $=1472$.

TCP Request/Response: tcp_rr_script is an implementation of the request/response benchmark over TCP. The request/response sizes are varied with the local/remote send and receive socket buffer sizes of each being the default of that particular system. With the local/remote send and receive socket buffer sizes remaining constant (the default), the output shows performance (transactions/s) as a function of request/response sizes (bytes). For the results in Table 10: send \& recv socket $=$ default bytes, request/resp. size $=1 / 1$.

UDP Request/Response: udp_rr_script is an implementation of the request/response benchmark over UDP. The request/response sizes are varied with the local/remote send and receive socket buffer sizes of each being the default of that particular system. With the local/remote send and receive socket buffer sizes remaining constant (the default), the output shows performance (transactions/s) as a 
functions of request/response sizes (bytes). For the results inTable 10: send \& recv socket $=$ default bytes, request/resp. size $=1 / 1$.

\begin{tabular}{|l|c|c|c|}
\hline System & Network & $\begin{array}{c}\text { TCP } \\
\text { request } \\
\text { response }\end{array}$ & $\begin{array}{c}\text { UDP } \\
\text { request } \\
\text { response }\end{array}$ \\
\hline DAISy systems & & & \\
FreeBSD/i586 (p5-90) & 10baseT & 1331 & 1659 \\
FreeBSD/i586 (p5-90) & 100baseT & 1638 & 2096 \\
\hline HEAT systems & & & \\
DEC AIpha & fddi & 1772 & 1937 \\
HP 9000/735 & fddi & 2423 & 2473 \\
SGI IRIX & fddi & 993 & 34 \\
\hline
\end{tabular}

Table 10. tcp_ח_script, udp_r_script results (transactions/s).

\subsubsection{Imbench suite (IPC Bandwidth)}

lmbench addresses the performance issues of

interprocess communication bandwidth with the

TCP bandwidth micro-benchmark. The results

shown in Table 11 are from the DAISy and

HEAT systems and from McVoy and Staelin's

Imbench draft [11].

\begin{tabular}{|lcc|}
\hline System & Network & $\begin{array}{c}\text { TCP (bw_tcp) } \\
\text { remote host }\end{array}$ \\
\hline SGI PowerChallenge & hppi & 79.3 \\
Sun Ultra 1 & 100baseT & 9.5 \\
HP 9000/735 & fddi & 8.8 \\
FreeBSD/1586 & 100baseT & 7.9 \\
SGI Indigo 2 & 10baseT & 0.9 \\
HP 9000/735 & 10baseT & 0.9 \\
Linux/1586@90Mhz & 10baseT & 0.7 \\
\hline DAISy systems & & \\
FreeBSD/1586 (p5-90) & 10baseT & 0.76 \\
FreeBSD/1586 (p5-90) & 100baseT & 6.26 \\
\hline \hline HEAT systems & & \\
DEC Alpha & fddi & 9.76 \\
HP 9000/735 & fddi & 9.02 \\
IBM RS6000 & fddi & 4.54 \\
SUN SS10 & fddi & 0.76 \\
SGI IRIX & fddi & 4.84 \\
\hline
\end{tabular}

Table 11. TCP bandwidth results (MB/s). bw_tcp (Table 11), the TCP micro-benchmark,

is a client/server program that moves $3 \mathrm{M}$ bytes

of data over a TCP/IP socket. The sockets are

configured to use the largest receive/send

buffers that the OS will allow.

\subsubsection{Imbench suite (IPC Latency)}

The cost of communicating between processes or IPC overhead consists of the time required to execute a system call and the time to move the data between processes. The lmbench suite implements both TCP and UDP latency IPC micro-benchmarks. The results shown in Table 12 are from the DAISy and HEAT systems and are compared to figures reported in [11].

\begin{tabular}{|c|c|c|c|c|c|}
\hline System & Network & $\begin{array}{l}\text { TCP } \\
\text { local } \\
\text { host }\end{array}$ & $\begin{array}{c}\text { remote } \\
\text { nost }\end{array}$ & $\begin{array}{l}\text { UDP } \\
\text { local } \\
\text { host }\end{array}$ & $\begin{array}{c}\text { remote } \\
\text { host }\end{array}$ \\
\hline $\begin{array}{l}\text { Sun Ultra1 } \\
\text { FreeBSDfi586 } \\
\text { SGI Indigo2 } \\
\end{array}$ & $\begin{array}{c}\text { 100baset } \\
\text { 100baseT } \\
\text { 10baseT }\end{array}$ & $\begin{array}{l}162 \\
256 \\
278 \\
\end{array}$ & $\begin{array}{l}280 \\
365 \\
543 \\
\end{array}$ & $\begin{array}{l}197 \\
212 \\
313\end{array}$ & $\begin{array}{l}308 \\
304 \\
602 \\
\end{array}$ \\
\hline $\begin{array}{l}\text { DAISy } \\
\text { systems } \\
\text { FreeBSD/i586 } \\
\text { (p5-90) } \\
\text { FreeBSD/1586 } \\
\text { (p5-90) }\end{array}$ & $\begin{array}{c}\text { 10base } 2 \\
\text { 100baseT }\end{array}$ & $\begin{array}{l}407 \\
442\end{array}$ & $\begin{array}{l}731 \\
572\end{array}$ & $\begin{array}{l}340 \\
378\end{array}$ & $\begin{array}{l}615 \\
470\end{array}$ \\
\hline \multicolumn{6}{|l|}{ HEAT systems } \\
\hline $\begin{array}{l}\text { DEC Alpha } \\
\text { HP } 9000 / 735\end{array}$ & $\begin{array}{l}\text { foddi } \\
\text { fddi }\end{array}$ & $\begin{array}{l}386 \\
222\end{array}$ & $\begin{array}{l}567 \\
419\end{array}$ & $\begin{array}{l}412 \\
225\end{array}$ & $\begin{array}{c}1089 \\
403\end{array}$ \\
\hline IBM RS6000 & fddi & 1178 & 2033 & 936 & 1893 \\
\hline SUN SS10 & fddi & 495 & 1243 & 515 & 1293 \\
\hline
\end{tabular}

Table 12. IPC latency results (microsec).

TCP: TCP connections are typically used in

low bandwidth latency sensitive applications.

TCP latency is measured by having a server 
process which waits for connections and a client process that connects to the server. The benchmark passes a token back and forth between the two processes through a TCP socket and measures the round trip time

UDP: UDP sockets are an alternative to TCP

sockets. UDP messages are commonly used in client server applications. UDP latency is measured by having a server process which waits for connections and a client process that connects to the server. The benchmark passes a token back and forth between two processes through a UDP socket and measures the round trip time

\subsubsection{Switch performance}

The following measurement uses a modified version of the lmbench TCP latency microbenchmark. Specifically, all function calls were replaced with macros in order to minimize overhead. The TCP latency of DAISy's 28115 LattisSwitch was measured using this code as follows. First, two nodes were connected to the frame switch and a request response latency was measured. The test was repeated, this time connecting both nodes together directly using a crossover cable. The difference between the two latencies is attributed to the overhead incurred by sending packets through the switch. The results show a switch latency of 13.74 micro seconds.

$$
\begin{aligned}
& \text { LAT TCP } \\
& 571.95 \text { us w/switch } \\
& 558.21 \text { us point-to-point } \\
& 13.74 \text { us latency through switch }
\end{aligned}
$$

\subsubsection{Discussion}

For the Netperf suite, a measure of the one-way latency for communication between two workstations is obtained by dividing the request/response time by two. In this case, the performance of DAISy systems was roughly comparable to that of the FDDI connected HEAT systems.

For the Imbench suite IPC bandwidth tests, in both the $10 \mathrm{Mb}$ and $100 \mathrm{Mb}$ network tests the DAISy cluster achieved slightly better than $50 \%$ of available bandwidth through the interface.

The 100Mb DAISy network lagged significantly behind the performance achieved from the FDDI network connected to each HEAT cluster node. The cause of this performance lag is due to the memory bandwidth of motherboards used in the DAISy cluster. Using Triton PCI chipset based motherboards that support Pipelined Burst SRAM, current Pentium 100 Mhz systems 
exceed $72 \mathrm{Mbit} / \mathrm{s}$ TCP throughput over Fast

Ethernet as measured by Netperf. The DAISy

nodes configured with P5-90 CPUs are not

powerful enough to drive the networking

hardware to the theoretical maximum.

\subsection{Message Passing Library Performance}

The application user on the DAISy cluster may use either of two different message passing libraries, PVM3 and MPI. Parallel application performance using message passing libraries can be strongly affected by the performance of the message passing libraries. This

performance may be characterized as having a message passing bandwidth and latency. The PVM3 and MPI libraries as used on the DAISy cluster use TCP as the underlying network protocol for node to node communication. In an efficient message passing library

implementation the performance of the library communication routines should be close to that of the underlying protocol.

\subsection{PVM3}

PVM3is a popular message passing library available from NETLIB[14]. The design of this library is intended to facilitate heterogeneous network computing, and thus is designed to ensure compatibility of messages passing operations across diverse platforms. However, a goal of DAISy was to construct the highest performing workstation cluster for the least cost. To this end, the design goals for DAISy consider only the homogeneous cluster of Pentium workstations. Thus certain optimizations were made. In particular, the RouteDirect option, which specifies point to point connections between message passing nodes, (and TCP transport) was used for the following measurements.

The PVM timing example is a simple program used to measure PVM message passing bandwidth and latency under PVM. It is a part of the example programs that are included in the PVM distribution. Table 13 shows the results from both the DAISy and HEAT clusters.

\begin{tabular}{|l|c|c|}
\hline System & Network & $\begin{array}{c}\text { PVM timing } \\
\text { ex. }\end{array}$ \\
\hline \hline DAISy systems & & \\
FreeBSD/1586 (p5-90) & 10baseT & 0.695 \\
FreeBSD/1586 (p5-90) & 100baseT & 5.284 \\
\hline \hline HEAT systems & & \\
DEC Alpha & fddi & 8.242 \\
HP 9000/735 & fddi & 9.5 \\
SGI IRIX & fddi & 6.66 \\
\hline
\end{tabular}

Table 13. PVM timing example results (avg bytes/usec). 


\subsection{Application Parallel Performance}

To verify the viability of commodity

workstation clusters, DAISy has been used to

perform various parallel computations. The

NAS Parallel Benchmarks (PVM [17] and MPI

[18] versions) along with a Parallel Seismic

Inverse Problem have demonstrated DAISy's

cost effectiveness.

\subsubsection{NAS Parallel Benchmarks 1.0, PVM version}

The NAS Parallel Benchmarks 1.0 (NPB 1.0)

consisted of eight benchmark problems. Five of

these were kernel benchmarks and three were

simulated computational fluid dynamics (CFD)

applications. We obtained the PVM versions of

the NPB1.0. Unfortunately, only one of the

eight benchmarks were able to run in class A

mode on the DAISy cluster. This was the

"embarrassingly parallel" benchmark EP. As a

note, a few of the recently published NPB 2.0

benchmarks running under MPI have run

successfully on DAISy and the results are

described in the next section.

Kernel EP: Briefly, Kernel EP executes $2^{\wedge} 28$ iterations of a loop in which a pair of random numbers are generated and tested for whether Gaussian random deviates can be made from them according to a specific scheme. The

number of pairs of the Gaussians in 10

successive square annuli are tabulated. The

pseudorandom number generator used in this ,

and in all NAS benchmarks which call for

random numbers, is of the linear congruential

recursion type. This kernel is viewed and

named as an "embarrassingly parallel"

application. In other words, improved

throughput rather than turn around time. Based

on the partitionability of the problem, no data or functional dependencies are incurred, and there is little or no communication between processors.

\begin{tabular}{|c|c|}
\hline $\begin{array}{c}\text { size }=2^{\wedge} 28 \\
\text { \# of processors }\end{array}$ & $\begin{array}{c}\text { Benchmark time }(\mathrm{sec}) \\
\text { p5-90, 100Mb/s sw }\end{array}$ \\
\hline \hline 15 & 287.97 \\
14 & 307.83 \\
13 & 331.45 \\
12 & 358.17 \\
11 & 391.82 \\
10 & 430.70 \\
9 & 481.27 \\
8 & 540.89 \\
7 & 616.46 \\
6 & 718.62 \\
5 & 865.28 \\
4 & 1076.66 \\
3 & 1440.13 \\
2 & 2152.87 \\
1 & 4304.07 \\
\hline
\end{tabular}

Table 14. Kernel EP results (sec)

Table 14 shows the scalability of the EP Kernel benchmark on the DAISy cluster. Note that the time it takes to execute the benchmark on one 
processor is almost exactly fifteen times slower than it would be to execute the benchmark on 15 processors. Hence, "embarrassingly parallel".

Table 15 shows the results from the DAISy and HEAT clusters using 8 nodes each.

\begin{tabular}{|l|c|c|}
\hline System & Network & Kernel EP \\
\hline DAISy systems & & \\
FreeBSD $/ 586(p 5-90)$ & 10baseT & 537 \\
FreeBSD $/ 586(p 5-90)$ & 100baseT & 541 \\
\hline \hline HEAT systems & & \\
DEC Alpha & fddi & 408 \\
IBM RS6K & fddi & 775 \\
SG! IRIX & fddi & 1193 \\
\hline
\end{tabular}

Table 15. Kernel EP results (sec).

\subsubsection{NAS Parallel Benchmarks 2.0, MPI versions}

NAS Parallel Benchmarks (NPB) 2.0 [19]

currently includes five of the original eight

benchmark problems, two of which are kernel

benchmarks (FT and MG) and three which are

computational fluid dynamics (CFD)

application benchmarks (LU, SP, and BT).

Results were obtained for the CFD application

benchmarks. The benchmarks are based on

FORTRAN 77 and the MPI message passing

standard. Table 16 shows the various problem

sizes for the NAS parallel benchmarks. DAISy

runs the Class A problem size. Table 17 shows

the standard operation count for the individual benchmarks with a Class A problem size and

the MFLOPS results for the DAISy cluster, with

the CRAY Y-MP/1 being the standard.

\begin{tabular}{|lccc|}
\hline Benchmark Code & Class A & Class B & Class C \\
\hline Embarrassingly Parallel & $2^{\wedge} 28$ & $2^{\wedge} 30$ & $2^{\wedge} 32$ \\
(EP) & & & \\
Multigrid (MG) & $256^{\wedge} 3$ & $256^{\wedge} 3$ & $512^{\wedge} 3$ \\
Conjugate Gradient & 14000 & 75000 & 150000 \\
(CG) & & & \\
3-D FFT PDE (FT) & $256^{\wedge} 2 \times 128$ & $512 \times 256^{\wedge} 2$ & $512^{\wedge} 3$ \\
Integer Sort (IS) & $2^{\wedge} 23$ & $2^{\wedge} 25$ & $2^{\wedge} 27$ \\
LU Solver (LU) & $64^{\wedge} 3$ & $102^{\wedge} 3$ & $162^{\wedge} 3$ \\
Pentadiagonal Solver & $64^{\wedge} 3$ & $102^{\wedge} 3$ & $162^{\wedge} 3$ \\
(SP) & & & \\
Block Tridiagonal & $64^{\wedge} 3$ & $102^{\wedge} 3$ & $162^{\wedge} 3$ \\
Solver (BT) & & & \\
\hline
\end{tabular}

Table 16. NAS Parallel Benchmarks Problem Sizes. From

D. Bailey, T. Harris, W. Saphir, R. van der Wijngaart, A.

Woo, and M. Yarrow's "The NAS Parallel Benchmarks 2.0"

[1995].

\begin{tabular}{|c|c|c|c|c|c|}
\hline \multirow[b]{2}{*}{ Name } & \multirow[b]{2}{*}{$\begin{array}{l}\text { Nominal } \\
\text { Size, } \\
\text { Class A }\end{array}$} & \multicolumn{2}{|c|}{ CRAY Y-MP/1 } & \multicolumn{2}{|c|}{ p5-90/16 100baseT } \\
\hline & & $\begin{array}{c}\text { Operation } \\
\text { Count } \\
\left(x 10^{\wedge} 9\right)\end{array}$ & MFLOPS & $\begin{array}{c}\text { MFLOPS } \\
\text { total }\end{array}$ & $\begin{array}{c}\text { MFLOPS } \\
\text { per } \\
\text { process }\end{array}$ \\
\hline (EP) & $2^{\wedge} 28$ & 26.68 & 211 & $\overline{\mathrm{NA}}$ & $\overline{\mathrm{NA}}$ \\
\hline (MG) & $256^{\wedge} 3$ & 3.905 & 176 & NA & NA \\
\hline (CG) & 14000 & 1.508 & 127 & NA & NA \\
\hline$(\mathrm{FT})$ & $\begin{array}{c}256^{\wedge} 2 \times 12 \\
8\end{array}$ & 5.631 & 196 & NA & NA \\
\hline (IS) & $2^{\wedge} 23$ & 0.7812 & 68 & NA & NA \\
\hline$(L U)$ & $64^{\wedge} 3$ & 64.57 & 194 & 56.01 & 3.5 \\
\hline$(\mathrm{SP})$ & $64^{\wedge} 3$ & 102 & 216 & 20.5 & 1.28 \\
\hline (BT) & $64^{\wedge} 3$ & 181.3 & 229 & 73.77 & 4.61 \\
\hline
\end{tabular}

Table 17. NAS Parallel Benchmarks Standard Operation Counts. From S. Saini, and D. H. Baile's "NAS Parallel Benchmark Results" [1995].

LU is a simulated CFD application which uses symmetric successive over-relaxation (SSOR) to solve a block lower triangular-block upper triangular system of equations resulting from an un-factored implicit finite-difference discretization of the Navier-Stokes equations in three dimensions. SP and BT are simulated 
CFD applications that solve systems of

equations resulting from an approximately

factored implicit finite-difference discretization

of the Navier-Stokes equations. BT solves

block-tridiagonal systems of $5 \times 5$ blocks; SP

solves scalar pentadiagonal systems resulting

from full diagonalization of the approximately

factored scheme.

\section{Application Benchmark (LU): The LU}

benchmark code requires a power-of-two

number of processors. A 2-D partitioning of the grid onto processors occurs by halving the grid repeatedly in the first dimensions, alternately $x$ and then $y$, until all power-of-two processors are assigned, resulting in vertical pencil-like grid partitions on the individual processors. The ordering of point based operations constituting the SSOR procedure proceeds on diagonals which progressively sweep from one comer on a given $z$ plane to the opposite corner of the same $z$ plane, thereupon proceeding to the next $z$ plane. Communication of partition boundary data occurs after completion of computational on all diagonals that contact an adjacent partition. This constitutes a diagonal pipelining method and is called a "wavefront" method. It results in a relatively large number of small communications of 5 words each. Table 18 shows the approximate sustained performance per dollar of DAISy and various systems for the Class A LU benchmark. Results for systems other than DAISy taken from [19].

\begin{tabular}{|c|c|c|c|c|c|c|c|}
\hline Computer System & \# of Proc. & Memory & $\begin{array}{c}\text { Time in } \\
\text { seconds }\end{array}$ & $\begin{array}{l}\text { Ratio to } \\
\text { CRAY Y- } \\
\text { MP/1 }\end{array}$ & $\begin{array}{l}\text { List Price } \\
\text { Million } \\
\text { Dollars } \\
\end{array}$ & $\begin{array}{c}\text { Performance } \\
\text { per Million } \\
\text { Dollars }\end{array}$ & Date \\
\hline CRAY Y-MP & $\overline{1}$ & $\overline{\text { NA }}$ & $\overline{333.5}$ & 1 & $\overline{\mathrm{NA}}$ & $\overline{\mathrm{NA}}$ & Aug-92 \\
\hline Convex SPP1000 & 32 & $4 \mathrm{~GB}$ & 126 & 2.65 & 2.5 & 1.06 & Mar-95 \\
\hline CRAY J916 & 16 & $2 \mathrm{~GB}$ & 47.59 & 7.01 & 1.05 & 6.67 & Jul-95 \\
\hline CRAY T3D & 1024 & $64 \mathrm{MB} / \mathrm{PE}$ & 7.09 & 47.04 & 3.6 & 13.07 & Mar-95 \\
\hline $\begin{array}{l}\text { DEC Alpha Server } \\
84005 / 300\end{array}$ & 12 & $2 \mathrm{~GB}$ & 79.13 & 4.21 & 0.718 & 5.87 & Oct-95 \\
\hline $\begin{array}{l}\text { IBM RS/6000 SP } \\
\text { Wide-node } 1(67 \mathrm{MHz})\end{array}$ & 128 & $128 \mathrm{MB} / \mathrm{PE}$ & 15.2 & 21.94 & 5.08 & 4.32 & Mar-95 \\
\hline $\begin{array}{l}\text { IBM RS/6000 SP } \\
\text { Wide-node2 }(77 \mathrm{MHz})\end{array}$ & 64 & $128 \mathrm{MB} / \mathrm{PE}$ & 19.2 & 17.37 & 5.74 & 3.03 & Oct-95 \\
\hline $\begin{array}{l}\text { IBM RS/6000 SP } \\
\text { Thin-node2 (67MHz) }\end{array}$ & 128 & 64MB/PE & 15.9 & 20.97 & 3.48 & 6.03 & Mar-95 \\
\hline SGI PC XL $(75 \mathrm{MHz})$ & 16 & $2 \mathrm{~GB}$ & 65.3 & 5.11 & 0.895 & 5.71 & Jun-94 \\
\hline SGI PC XL (90MHz) & 16 & $2 \mathrm{~GB}$ & 65.9 & 5.06 & 1.02 & 4.96 & May-95 \\
\hline DAISy & 16 & $64 \mathrm{MB} /$ node & 2897.49 & 0.12 & 0.06 & 1.92 & Nov-95 \\
\hline
\end{tabular}

Table 18. Approximate sustained performance per dollar for Class A LU benchmark. From S. Saini, and D. H. Baile's "NAS Parallel Benchmark Results" [1995]. 
Application Benchmark (SP and BT): The SP

and BT algorithms have a structure similar to

the LU algorithm: Each solves three sets of

uncoupled systems of equations, first in the $x$,

then in the $y$, and finally in the $z$ direction.

These systems are scalar pentadiagonal in the

SP code, and block tridiagonal with $5 \times 5$ blocks

in the BT code.

The implementations of the SP and BT solve

these systems using a multi-partition scheme.

In the multi-partition algorithm each processor

is responsible for several disjoint sub-blocks of

points ("cells") of the grid. The cells are

arranged such that for each direction of the line

solve phase the cells belonging to a certain

processor will be evenly distributed along the direction of solution. This allows each

processor to perform useful work throughout a

line solve, instead of being forced to wait for

the partial solution to a line from another

processor before beginning work. Additionally,

the information from a cell is not sent to the

next processor until all sections of linear

equation systems handled in this cell have been

solved. Therefore, the granularity of

communications is kept large and fewer

messages are sent.

Both the SP. and BT codes require a square

number of processors. Table 19 and Table 20

show the approximate sustained performance

per dollar of DAISy and various systems for

Class A SP and BT benchmarks respectively.

\begin{tabular}{|c|c|c|c|c|c|c|c|}
\hline Computer System & $\begin{array}{l}\text { \# of } \\
\text { Proc. }\end{array}$ & Memory & $\begin{array}{l}\text { Time in } \\
\text { seconds }\end{array}$ & $\begin{array}{c}\text { Ratio to } \\
\text { CRAY Y- } \\
\text { MP/1 }\end{array}$ & $\begin{array}{l}\text { List Price } \\
\text { Million } \\
\text { Dollars }\end{array}$ & $\begin{array}{c}\text { Perform } \\
\text { ance per } \\
\text { Million } \\
\text { Dollars }\end{array}$ & Date \\
\hline CRAY Y-MP & 7 & NA & 471.5 & $\overline{1}$ & NA & NA & Aug-92 \\
\hline Convex SPP1000 & 64 & $4 \mathrm{~GB}$ & 102 & 4.62 & 2.5 & 1.84 & Mar-95 \\
\hline CRAY J916 & 16 & $2 \mathrm{~GB}$ & 77.54 & 6.08 & 1.05 & 5.79 & Jul-95 \\
\hline CRAY T3D & 1024 & $64 \mathrm{MB} / \mathrm{PE}$ & 5.41 & 87.15 & 3.6 & 24.21 & Mar-95 \\
\hline $\begin{array}{l}\text { DEC Alpha Server } \\
84005 / 300\end{array}$ & 12 & $2 \mathrm{~GB}$ & 102.75 & 4.59 & 0.718 & 6.39 & Oct-95 \\
\hline $\begin{array}{l}\text { IBM RS/6000 SP } \\
\text { Wide-node1 }(67 \mathrm{MHz})\end{array}$ & 128 & $128 \mathrm{MB} / \mathrm{PE}$ & 18.7 & 25.21 & 5.08 & 4.96 & Mar-95 \\
\hline $\begin{array}{l}\text { IBM RS/6000 SP } \\
\text { Wide-node2 }(77 \mathrm{MHz})\end{array}$ & 64 & $128 \mathrm{MB} / \mathrm{PE}$ & 26.46 & 17.82 & 5.74 & 3.10 & Oct-95 \\
\hline $\begin{array}{l}\text { IBM RS/6000 SP } \\
\text { Thin-node2 }(67 \mathrm{MHz})\end{array}$ & 128 & 64MB/PE & 20.6 & 22.89 & 3.48 & 6.58 & Mar-95 \\
\hline SGI PC XL (75MHZ) & 16 & $2 \mathrm{~GB}$ & 67.2 & 7.02 & 0.895 & 7.84 & Jun-94 \\
\hline SGI PC XL (90MHz) & 16 & $2 \mathrm{~GB}$ & 63.18 & 7.46 & 1.02 & 7.32 & May-95 \\
\hline DAISy & 16 & $64 \mathrm{MB} /$ node & 3883.83 & 0.12 & 0.06 & 2.02 & Nov-95 \\
\hline
\end{tabular}

Table 19. Approximate sustained performance per dollar for Class A SP benchmark. From S. Saini, and D. H. Bailey's "NAS Parallel Benchmark Results" [1995]. 


\begin{tabular}{|c|c|c|c|c|c|c|c|}
\hline Computer System & $\begin{array}{l}\text { \# of } \\
\text { Proc. }\end{array}$ & Memory & $\begin{array}{l}\text { Time in } \\
\text { seconds }\end{array}$ & $\begin{array}{c}\text { Ratio to } \\
\text { CRAY Y- } \\
\text { MP/1 }\end{array}$ & $\begin{array}{c}\text { List Price } \\
\text { Million } \\
\text { Dollars }\end{array}$ & $\begin{array}{c}\text { Performance } \\
\text { per Million } \\
\text { Dollars }\end{array}$ & Date \\
\hline CRAY Y-MP & 1 & $\overline{\mathrm{NA}}$ & 792.4 & 1 & NA & $\overline{\mathrm{NA}}$ & Aug-92 \\
\hline Convex SPP 1000 & 64 & $4 \mathrm{~GB}$ & 78 & 10.16 & 1.25 & 8.13 & Mar-95 \\
\hline CRAY J916 & 16 & $2 \mathrm{~GB}$ & 98.8 & 8.02 & 1.05 & 7.64 & Jul-95 \\
\hline CRAY T3D & 1024 & $64 \mathrm{MB} / \mathrm{PE}$ & 4.56 & 173.77 & 3.6 & 48.27 & Mar-95 \\
\hline $\begin{array}{l}\text { DEC Alpha Server } \\
84005 / 300\end{array}$ & 12 & $2 \mathrm{~GB}$ & 103.47 & 7.66 & 0.718 & 10.67 & Oct-95 \\
\hline $\begin{array}{l}\text { IBM RS/6000 SP } \\
\text { Wide-node }(67 \mathrm{MHz})\end{array}$ & 128 & $\begin{array}{c}128 \\
M B / P E\end{array}$ & 20.1 & 39.42 & 5.08 & 7.76 & Mar-95 \\
\hline $\begin{array}{l}\text { IBM RS/6000 SP } \\
\text { Wide-node } 2(77 \mathrm{MHz})\end{array}$ & 64 & $\begin{array}{c}128 \\
\mathrm{MB} / \mathrm{PE}\end{array}$ & 29.01 & 27.31 & 5.74 & 4.76 & Oct-95 \\
\hline $\begin{array}{l}\text { IBM RS/6000 SP } \\
\text { Thin-node2 (67MHz) }\end{array}$ & 128 & 64MB/PE & 20.8 & 38.10 & 3.48 & 10.95 & Mar-95 \\
\hline SGI PC XL $(75 \mathrm{MHz})$ & 16 & $2 \mathrm{~GB}$ & 91.8 & 8.63 & 0.895 & 9.64 & Jun-94 \\
\hline SGI PC XL (9OMHz) & 16 & $2 \mathrm{~GB}$ & 80.2 & 9.88 & 1.02 & 9.69 & May-95 \\
\hline DAISy & $\overline{16}$ & $\begin{array}{c}64 \\
\text { MB/node }\end{array}$ & 2641.61 & 0.30 & 0.06 & 5.00 & Nov-95 \\
\hline
\end{tabular}

Table 20. Approximate sustained performance per dollar for Class A BT benchmark. From S. Saini, and D. H. Bailey's "NAS Parallel Benchmark Results" [1995].

Again, data for systems other than DAISy is taken from [19].

\subsubsection{Discussion of NPB Results}

The evaluation of the performance of the

DAISy cluster on the three NPB application benchmarks LU, BT, and SP is complicated by the source and intentions of the data which is being examined. First, the results reported in [19] were obtained under NPB 1.0 rules. However, the data reported for DAISy was obtained under NPB 2.0 rules. The difference in the two sets of rules is essentially that NPB 1.0 rules allow intensive optimization of codes in order to assess the absolute maximum performance obtainable on the algorithm from a particular architecture, while NPB 2.0 rules are intended to determine the performance of a parallel architecture on a portable parallel code using MPI as the message passing standard.

The codes run on DAISy were modified only to the extent needed to run; i.e., no algorithmic optimizations were made In view of these differences in the source of data, it is impressive that NPB SP, BT, and LU implementations run on DAISy have price/performance with effectively unmodified, portable MPI message passing codes that exceed that of highly optimized codes on several architectures, and is competitive with many, including shared memory architectures. This comparison between NPB 1.0 results for other systems and NPB 2.0 results from DAISy is necessitated by 
the lack of NPB 2.0 data at the time this

analysis was performed. It is expected that

DAISy will exhibit much better comparative

performance against most other systems when

NPB 2.0 data becomes available for them.

\subsubsection{Parallel Seismic Inverse Problem}

The DAISy cluster has been used to calculate an inverse problem in seismic tomography. The project goal [19] is to demonstrate a parallel seismic inverse code that runs scalably on inexpensive IBM compatible platforms, incorporating a modular design that separates the parallel algorithm from the specific model used for seismic imaging. The seismic data generated by means of impacts on the earth's surface, consists of timings between generation and reception. The data can be inverted through a tomographic scheme to give a threedimensional picture of the local rock velocity.

The algorithm is a hybrid of bisection ray tracing and a P-wave Huygens' principle approach and parallelizes in an embarrassingly parallel manner. The algorithm has an adjustable parameter that controls the resolution of the resulting 3D velocity distribution. High resolutions will require $\sim 1$ sec between communications, while lower resolutions

require $\sim .01 \mathrm{sec}$. Though, this code is

embarrassingly parallel, it is ideal to test the

sensitivity of the cluster to network latency.

Figure 3 shows a three-dimensional rendered image of the subterranean galleries of the "Lucky Friday" silver mine located in Northern

Idaho. For acceptable tomographic feature prediction $1 \mathrm{sec}$ to $.1 \mathrm{sec}$ is required per task (on the DAISy $90 \mathrm{MHz}$ Pentium). This is useful as a check on the inverse model because the topography of the mine tunnels are measured.

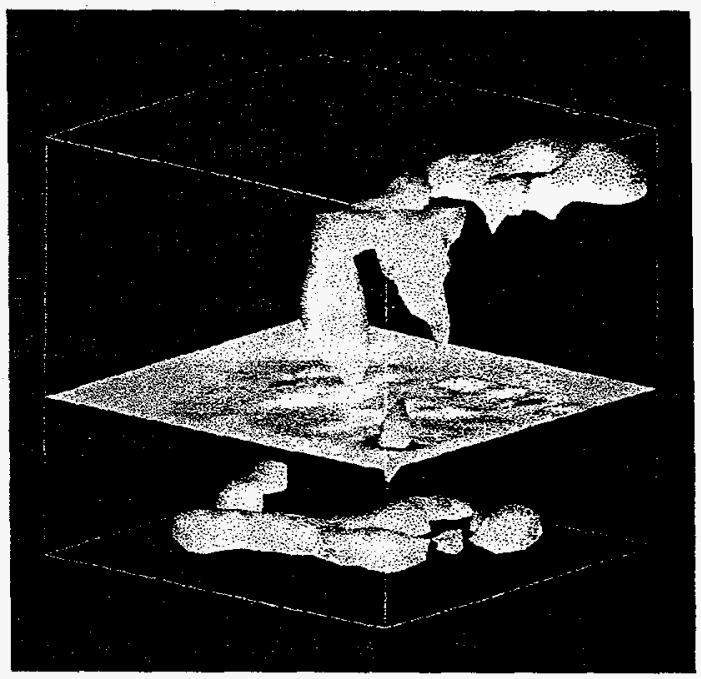

Figure 3. Parallel Seismic Inverse Model. This is the tomographic rendering from seismic data for the "Lucky Friday" silver mine in Northern Idaho. The gold features accurately predict the known locations of the mine galleries. The blue plane is an orthogonal slice through the observation volume. Colors on this plane indicate the effective "sound" velocity of the rock: red is faster, blue is slower.

Figure 4 shows the execution time for the parallel seismic inverse model on various 
platforms. All runs used the same source code and the GNU $\mathrm{C}++$ compiler $(\mathrm{G}++)$ for the native OS without optimization. The PC cluster performs admirably against the considerably more costly workstations.

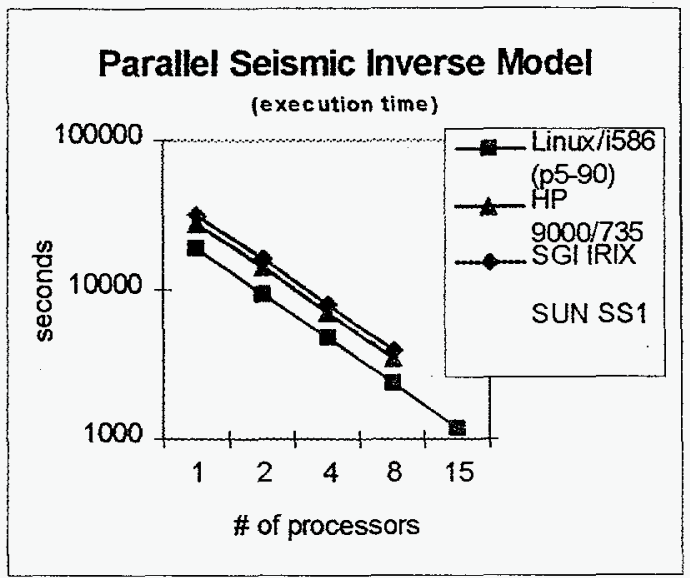

Figure 4. Execution time for Parallel Seismic Inverse Model

\section{$5 \quad$ Summary}

Workstation clusters were originally developed as a way to leverage the better cost basis of UNIX workstations to perform computations previously handled only by relatively more expensive supercomputers. Commodity workstation clusters take this evolutionary process one step further by replacing equivalent proprietary workstation functionality with less expensive PC technology. As PC technology encroaches on proprietary UNIX workstation vendor markets, these vendors will see a

declining share of the overall market.

As technology advances continue, the ability to upgrade a workstations performance plays a large role in cost analysis. For example, a major upgrade to a typical UNIX workstation means replacing the whole machine. As major revisions to the UNIX vendor's product line come out, brand new systems are introduced. IBM compatibles, however, are modular by design, and nothing need be replaced except the components that are truly improved. The DAISy cluster, for example, is about to undergo a major upgrade from $90 \mathrm{MHz}$ Pentiums to $200 \mathrm{MHz}$ Pentium Pros. All of the memory - the system's largest expense - and disks, power supply, etc., can be reused. As a result, commodity workstation clusters ought to gain an increasingly large share of the distributed computing market. 
[1] Donald J. Becker, Thomas Sterling, Daniel Savarese, Bruce Fryxell, Kevin Olsen, "Communication Overhead for Space Science Applications on the Beowulf Parallel Workstation", High Performance Distributed Computing Conference, August 1-4, 1995, http://cesdis.gstc.nasa.gov/linux/beowulf/hpdc95.html.

[2] Edward Solari and George Willse, "PCI Hardware and Software", Annabooks, San Diego, CA, 1994.

[3] http://www.freebsd.org.

[4] Linux Documentation Project, http://sunsite.unc.edu/mdw/linux.html.

[5] Al Geist, Adam Beguelin, Jack Dongarra, Weicheng Jiang, Robert Mancheck, Vaidy Sunderam, "PVM 3 User's Guide and Reference Manual”, Oak Ridge National Laboratory Technical Memorandum 12187, May, 1994.

[6] "MPI: A Message-Passing Interface Standard", Message Passing Interface Forum, May 5, 1994.

[7] Stephen S. Fried, "Pentium Optimization and Numeric Performance", Dr.Dobb's Journal, pp.18-29, January, 1995.

[8] Michael L. Schmit, "Pentium Processor Optimization Tools", Academic Press, Cambridge MA, 1995.

[9] J. J. Dongarra, J. Du Croz, I. S. Duff, and S. Hammarling, A Set of 3 Basic Linear Algebra Subprograms, ACM Trans. Math. Soft., 16 (1990), pp. 1-17.

[10] McCalpin, John D, "Memory Bandwidth and Machine Balance in Current High Performance Computers." Invited for submission to IEEE Technical Committee on Computer Architecture newsletter. To appear December 1995.

[11] L. McVoy, C. Staelin. "Imbench: Portable Tools for Performance Analysis", Proceedings of the USENIX Annual Technical Conference, San Diego, CA, 1996.

[12] R. Weicker, J. Reilly [1995]. "SPEC Frequently Asked Questions (FAQ) / SPEC Primer". WWW Tech. Doc., Web Page (December 15), Siemens Nixdorf, Paderborn/Germany, weicker.pad@sni.de, and (Intel) and R.Weicker, jwreilly@mipos2.intel.com. URL: http://hpwww.epfl.ch/bench/SPEC.FAQ.html.

[13] Jack J. Dongarra, "Performance of Various Computers Using Standard Linear Equations Software", University of Tennessee Computer Science Technical Report CS-90-85, February 24,1995 .

[14] http://www.netlib.org

[15] Tim Bray, Bonnie source code, 1990.

[16] Information Networks Division, Hewlett-Packard Company [1995], "Netperf: A Network Performance Benchmark, Revision 2.0", Tech. Rep. (February 15), Hewlett-Packard Company, URL http://www.cup.hp.com/netperf/NetperfPage.html. 
[17] S. White, A. Alund, and V.S. Sunderam, [199x]. "Performance of the NAS Parallel Benchmarks on PVM Based Networks", Tech. Rep., Department of Mathematics and Computer Science, Emory University, Atlanta, Georgia.

[18] D. Bailey, T. Harris, W. Saphir, R. van der Wijngaart, A. Woo, and M. Yarrow, [1995]. "The NAS Parallel Benchmarks 2.0", Tech. Rep. (December), NASA Ames Research Center, Moffett Field, California.

[19] Subhash Saini and David H. Bailey, "NAS Parallel Benchmark Results, 12-95", Report NAS95-021, Dec. 1995, NASA Ames Research Center, Moffett Field, CA.

[20] Rob Armstrong, [1994]. "Parallel Seismic Inverse Model". Tech. Demo. Handout (November), Sandia National Laboratories, Livermore, California.

\section{DISCLAIMER}

This report was prepared as an account of work sponsored by an agency of the United States Government. Neither the United States Government nor any agency thereof, nor any of their employees, makes any warranty, express or implied, or assumes any legal liability or responsibility for the accuracy, completeness, or usefulness of any information, apparatus, product, or process disclosed, or represents that its use would not infringe privately owned rights. Reference herein to any specific commercial product, process, or service by trade name, trademark, manufacturer, or otherwise does not necessarily constitute or imply its endorsement, recommendation, or favoring by the United States Government or any agency thereof. The views and opinions of authors expressed herein do not necessarily state or reflect those of the United States Government or any agency thereof. 